\title{
Extracellular signal-regulated kinase signaling-mediated induction and interaction of FOXO3a and p53 contribute to the inhibition of nasopharyngeal carcinoma cell growth by curcumin
}

\author{
JINGJING WU, QIN TANG, SHUNYU ZHAO, FANG ZHENG, YAN WU, GE TANG and SWEI SUNNY HAHN \\ Laboratory of Tumor Molecular Biology and Targeted Therapies of Chinese Medicine, University of Guangzhou \\ Traditional Chinese Medicine, Guangdong Provincial Hospital of Chinese Medicine, Guangzhou 510120, P.R. China
}

Received February 21, 2014; Accepted April 2, 2014

DOI: $10.3892 /$ ijo.2014.2420

\begin{abstract}
Curcumin, one of the main bioactive components extracted from a traditional Chinese medicinal herb, exhibits potent anticancer activity against many types of cancer cells including nasopharyngeal carcinoma (NPC). However, the detailed molecular mechanism underlying this is not clearly understood. In this study, we showed that curcumin significantly inhibited the growth of NPC cells in a doseand time-dependent manner as determined by MTT assays, while increasing apoptosis was also observed as measured by flow cytometry for the FITC-Annexin V and propidium iodide (PI) label and Hoechst 33258 staining. To further explore the potential mechanism, we showed that curcumin increased the phosphorylation of ERK1/2 but not p38 MAPK in a time-dependent manner, and induced protein expression of the tumor suppressors FOXO3a and p53 in a dose-dependent manner, which were not observed in the presence of PD98059, an inhibitor of ERK1/2. Furthermore, silencing of FOXO3a and p53 genes by siRNAs overcame the inhibitory effect of curcumin on cell proliferation. Silencing or blockade of p53 using siRNA or chemical inhibitor abrogated the effect of curcumin on expression of FOXO3a protein; silencing or overexpression of FOXO3a had no further effect on curcumin-induced p53 protein expression. Furthermore, blockade of ERK1/2 and exogenous expression of FOXO3a restored the effect of curcumin on growth of cells. Together, our studies show that curcumin inhibits growth and induces apoptosis of NPC cells through ERK1/2-mediated increase in the protein expression and interaction of $\mathrm{p} 53$ and FOXO3a.
\end{abstract}

Correspondence to: Professor Swei Sunny Hahn, Laboratory of Tumor Molecular Biology and Targeted Therapies of Chinese Medicine, Guangdong Provincial Hospital of Chinese Medicine, 4th Floor, Scientific Research Building, Neihuan West Road No. 55, University City, Panyu, Guangzhou, Guangdong 510006, P.R. China E-mail: swhan2010@live.com

Key words: curcumin, nasopharyngeal carcinoma cells, ERK1/2, FOXO3a, p53 p53 is upstream of FOXO3a, which form a regulatory loop that mediates the effect of curcumin. This study unveils a new mechanism by which curcumin inhibits the proliferation and induces apoptosis of human NPC cells.

\section{Introduction}

Nasopharyngeal carcinoma (NPC) is one of the most common cancers of the head and neck, particularly in southern China and Southeast Asia $(1,2)$. Though NPC patients are sensitive to radio/chemo-therapy, treatment failure remains high due to the development of local recurrence and distant metastasis (1). Epstein-Barr virus (EBV) infection, environmental factors and genetic susceptibility are associated with this malignancy (2). To date, the molecular mechanisms related to the progression and clinical outcome of NPC have not yet been fully understood. A combination of radiotherapy and adjuvant chemotherapy has been successful for certain NPC patients, but the 5 -year survival rate is only $50-60 \%$. There is almost no effective treatment for those who are resistant to radiotherapy and have tumor recurrence. Therefore, we need to further explore the pro-oncogenic pathways that lead to or promote NPC with the intention of identifying new targets for therapy and improving patient survival. In addition, understanding the molecular mechanisms and key regulatory factors exploitable in developing adjuvant therapies to augment currently available treatment protocols that allow decreased side-effects and toxicity without compromising therapeutic efficacy are also required. Recently, much attention has been focused on the use of traditional Chinese medicinal herbs in cancer prevention and therapy due to the pleiotropic effects of these agents on multiple carcinogenactivated oncogenic pathways, and their equally excellent safety profiles. Curcumin is one such potential candidate.

Curcumin, one of the main bioactive components extracted from a traditional Chinese medicinal herb, is associated with a variety of functions such as immunomodulatory, anti-inflammatory and anticancer activities (3). Substantial evidence has demonstrated that curcumin acts as a potential chemopreventive agent as well as a novel adjuvant treatment agent for head and neck malignancies via its effect on a variety of biological pathways (4). However, the detailed 
mechanisms by which this agent is involved in the inhibition of NPC cell growth and induction of apoptosis have not been well elucidated.

The p53 tumor suppressor is a major regulator of cell proliferation and apoptosis, which is activated in response to DNA damage and is mutated in $~ 50 \%$ of human cancers (5). As a tumor suppressor protein, p53 plays a pivotal role in regulating the cellular response to stress and damage signals, and loss of p53 functionality is common in more than 50\% of cancers (6). The role of p53 in the link of NPC has been reported, activation of $\mathrm{p} 53$ was involved in the radioresponse in NPC (7) and played an important role in the development of novel therapies for NPC treatment (8). The forkhead box, class $\mathrm{O}$ belongs to the family of mammalian forkhead transcription factors, including FOXO3a (or FKHRL1), FOXO1a (or FKHR), and FOXO4a (or AFX), which are regulated by growth factor receptor-induced activation of the phosphatidylinositol 3-kinase (PI3K)/AKT (or protein kinase B) signaling pathway (9). Studies in mammalian cells have shown that activation of FOXO3a by stimulated the expression of proteins that are involved in apoptosis (9) and cell cycle arrest (10) in different types of cells. FoxO3a is a transcription factor with known tumor suppressor activity and a conserved 110-amino acid DNA-binding domain and recognize two consensus DNA-binding sequences: 5'-TTGTTTAC-3' and 5'-(C/A)(A/C)AAA(C/T)AA-3' (11). Inhibition of FOXO3a expression promotes cell transformation, tumor progression and angiogenesis (12). Expression of FOXO3a was reported in NPC and considered as important prognostic marker in NPC (13).

In the present study, we investigated the potential mechanism by which curcumin inhibited NPC cell growth and induced apoptosis. Our results showed that extracellular signal-regulated kinase 1/2 (ERK1/2) signaling pathwaymediated increase in the protein expression and the interaction of FOXO3a and p53 contributed to the effect of curcumin on proliferation and apoptosis of NPC cells.

\section{Materials and methods}

Reagents. Curcumin was purchased from Sigma-Aldrich (Shanghai, China). RPMI-1640 and penicillin/streptomycin were purchased from Invitrogen (Carlsbad, CA, USA), fetal bovine serum (FBS) was purchased from HyClone (UT, USA). Monoclonal antibodies specific for p38 mitogen-activated protein kinase (p38 MAPK); ERK1/2 and their phosphor-forms, and FOXO3a and p53 were purchased from Cell Signaling Technology (Beverly, MA, USA). The GAPDH, p53 and FOXO3a monoclonal antibodies were obtained from Abcam Co (Burlingame, CA, USA). PVDF membrane was purchased from Millipore (USA). PD98059 (a special inhibitor of ERK1/2) was purchased from Merck Millipore (Darmstadt, Germany), MTT powder and pifithrin- $\alpha$ (a special inhibitor of p53) were purchased from Sigma-Aldrich (St. Louis, MO, USA). p53 and FOXO3a siRNAs were obtained from Santa Cruz (CA, USA). Lipofectamine 2000 reagent was purchased from Invitrogen (Shanghai, China). The FOXO3a-GFP and N1-GFP plasmids were kindly provided by Frank M.J. Jacobs (Rudolf Magnus Institute of Neuroscience, Department of Pharmacology and Anatomy, University Medical Center, Utrecht) and was reported previously (14). Annexin V-FITC Apoptosis Kit was purchased from Bestbio Co. (Shanghai, China).

Cell culture. Human NPC cell lines CNE2 and HNE2 were obtained from the Cell Line Bank at the Laboratory Animal Center of Sun Yat-sen University starting March, 2012 (Guangzhou, China). All cell lines have been tested and authenticated for absence of mycoplasma, genotypes, drug response, and morphology using a commercially available kit (Invitrogen, Shanghai, China) in the Laboratory Animal Center at Sun Yat-sen University in April, 2010. Cells were cultured in RPMI-1640 medium, supplemented with $10 \%$ fetal bovine serum (FBS), $5 \%$ glutamine, $100 \mathrm{U} / \mathrm{ml}$ penicillin and $100 \mathrm{mg} / \mathrm{ml}$ streptomycin. In all experiments, $60-70 \%$ of confluent cells were washed and incubated with curcumin, PD98059, pifithrin- $\alpha$ for the indicated time. Curcumin was dissolved in DMSO and the final concentration was $<1 \%$ (v/v) in all experiments.

MTT assay. Cell viability was analyzed by the MTT [3-(4, 5-dimethylthiazol-2-yl)-2, 5-diphenyl tetrazolium bromide] assay. Briefly, cells were seeded in 96-well plates at the density of $2 \times 10^{3}$ cells/well and were cultured with increased concentrations of curcumin for up to $72 \mathrm{~h}$, and then $10 \mu \mathrm{l}$ of $10 \mathrm{mg} / \mathrm{ml}$ MTT solution was added to each well for an additional $4 \mathrm{~h}$ according to the manufacturer's instructions (Promega, Shanghai, China). After centrifugation, $150 \mu 1$ dimethyl sulfoxide was added to the precipitate and the absorbance of the enzyme was measured at $570 \mathrm{~nm}$ using a Microplate Reader (Bio-Rad, Hercules, CA, USA). Cell growth rates (average absorbance of each treat group and non-treated group) were then calculated. All experiments were performed in triplicate and repeated at least three times.

Hoechst 33258 staining. Following treatment with curcumin at various concentrations for up to $48 \mathrm{~h}$, cells were washed twice with PBS and fixed in $1 \mathrm{ml}$ of $4 \%$ paraformaldehyde for $10 \mathrm{~min}$ at $4^{\circ} \mathrm{C}$. After washing twice with PBS, cells were stained with $100 \mu \mathrm{l}(10 \mu \mathrm{g} / \mathrm{ml})$ Hoechst 33258 (Sigma, St. Louis, MO, USA) in PBS for 15 min at room temperature in the dark, and then washed with PBS. Afterwards, the cells were mounted and examined under fluorescence microscopy (Olympus IX71, Tokyo, Japan). Apoptotic cells were identified by the condensation and fragmentation of their nuclei.

Flow cytometry. Cells ( $1 \times 10^{5}$ cells/dish) were seeded in $6-\mathrm{mm}$ culture plate and treated with increased doses of curcumin as indicated for up to $48 \mathrm{~h}$. The adherent and floating cells were both collected and resuspended in cold PBS for analysis. Cells were stained with Annexin V-FITC apoptosis detection kit to monitor apoptosis cells and propidium iodide (PI) to detect dead cells according to the instructions from the provider. Samples were analyzed on the FC500 Flow cytometry Systems (Beckman Coulter, USA).

Western blot analysis. After being treated with curcumin, cells were harvested and washed with ice-cold phosphate buffer, homogenized in 1X RIPA lysis buffer. Equal amounts of protein from whole cell lysates were solubilized in $2 \mathrm{X}$ SDS-sample buffer, separated on SDS-polyacrylamide gels. 

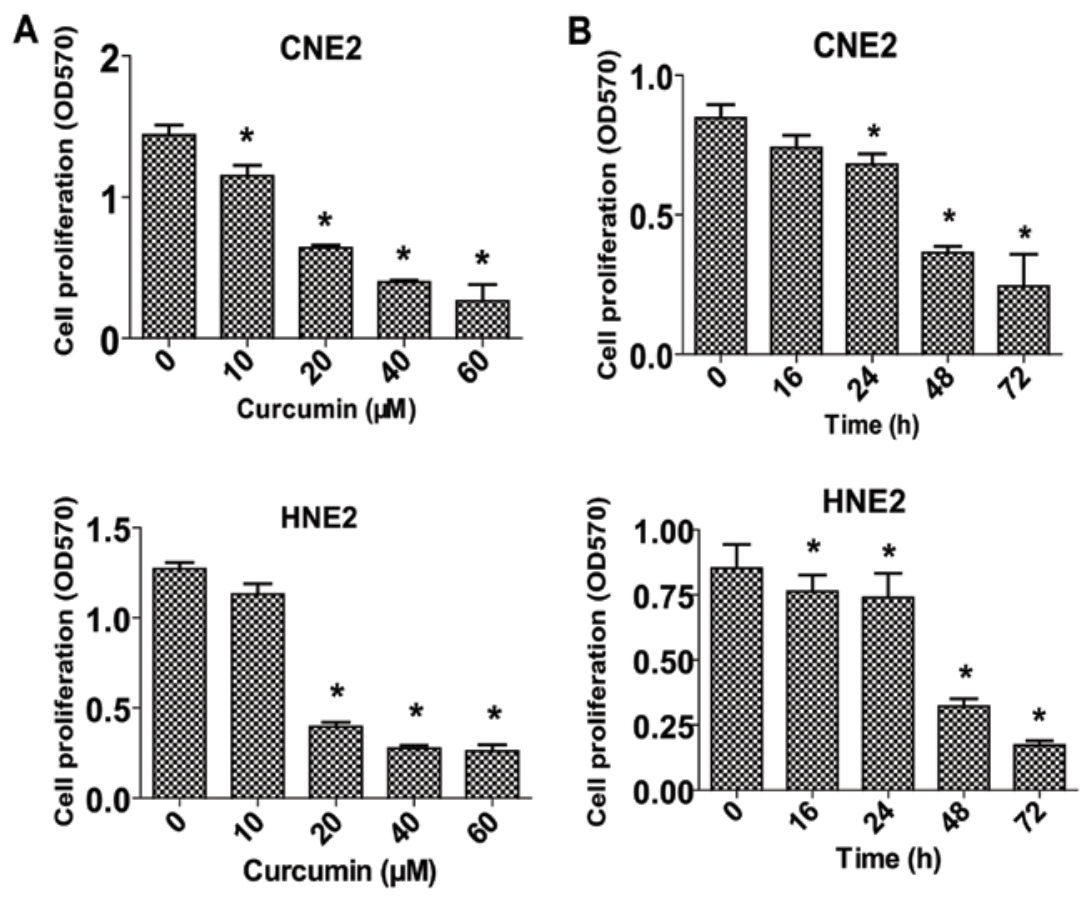

Figure 1. Curcumin inhibits proliferation of NPC cells. (A) Human CNE2 and HNE2 NPC cells were treated with increased concentrations of curcumin and indicated for $48 \mathrm{~h}$ (A) or at the concentration of $40 \mu \mathrm{M}$ for up to $72 \mathrm{~h}$ (B). Afterwards, cell proliferation was determined by MTT assays. The data are presented as mean \pm SD of three separate experiments. ${ }^{*} \mathrm{P}<0.05$ indicates significant difference as compared to the untreated control group.

The separated proteins were transferred onto nitrocellulose using a Bio-Rad Trans Blot semidry transfer apparatus for $1 \mathrm{~h}$ at $25 \mathrm{~V}$, blocked with Blotto with 5\% no-nfat dry milk and $0.1 \%$ Tween- 20 overnight at $4^{\circ} \mathrm{C}$, and washed with wash buffer. Blots were incubated with polyclonal antibodies against p38 MAPK, ERK1/2 and their phosphor-forms, and FOXO3a and p53 $(1: 1,000)$ overnight at $37^{\circ} \mathrm{C}$, washed and incubated with a secondary antibody raised against rabbit $\mathrm{IgG}$ conjugated to horseradish peroxidase (1:2,000, Sigma, Beijing, China) for $1 \mathrm{~h}$ at room temperature. The washed blots were transferred to freshly made ECL Prime (Pierce, Rockford, IL, USA) and exposed to X-ray film.

Treatment with p53 and FOXO3a small interfering RNAs. For the transfection procedure, cells were grown to $60 \%$ confluence, and p53 and FOXO3a siRNAs and control siRNA were transfected using the oligofectamine reagent (Invitrogen). CNE2 cells $\left(2 \times 10^{5}\right.$ cells $\left./ \mathrm{ml}\right)$ were seeded in RPMI-1640 medium without antibiotics overnight. After washing the cells with PBS, $2 \mathrm{ml}$ of media without antibiotics were added. Thereafter, $200 \mu \mathrm{l}$ of Lipofectamine 2000 complex was added into each plate. The cells were transfected with control or FoxO3a (FKHRL1) and p53 small interfering RNAs (siRNAs) for $24 \mathrm{~h}$ according to instructions from the manufacturer. At $24 \mathrm{~h}$ after transfection, medium was replaced for complete DMEM and cells were transfected with either exogenous FOXO3a gene using electroporated transfection method or treated with curcumin $40 \mu \mathrm{M}$ for an additional $24 \mathrm{~h}$ for other experiments.

Electroporated transfection assays. CNE2 cells $\left(1 \times 10^{7}\right.$ cells/ml) were transferred into conical tubes and centrifuged at 1,200 rpm for $5 \mathrm{~min}$. After centrifuging, the medium was removed and the cells were washed with $1 \mathrm{X}$ PBS, and centrifuged again at 1,200 rpm for $5 \mathrm{~min}$. Afterwards, the PBS was aspirated and added to Bio-Rad Gene Pulser electroporation buffer. After resuspending the cells, the desired N1-GFP or FoxO3a-GFP plasmid DNA at a final concentration of 10 to $20 \mu \mathrm{g} / \mathrm{ml}$ was added and the electroporation plate was put in the MXcell plate chamber. The electroporation conditions on the plates to deliver $160 \mathrm{~V} / 10 \mathrm{~ms}$ square wave were adjusted until reaching the optimum. The conditions were set and loaded onto the device Gene Pulser II Electroporation System (Bio-Rad, CA, USA). After electroporation was completed, the cells were transferred to a tissue culture plate. We typically transferred each $150 \mu$ l electroporation sample to a 6 -well tissue culture plate containing $2 \mathrm{ml}$ RPMI-1640. Cells were then incubated for $48 \mathrm{~h}$ at $37^{\circ} \mathrm{C}$, then treated with curcumin for an additional $24 \mathrm{~h}$.

Statistical analysis. Analysis of variance and Student's t-test were used to compare the values of the test and control samples. $\mathrm{P}<0.05$ was considered a statistically significant difference. SPSS software was used for all statistical analysis. The significance was evaluated by the paired t-test. All the experiments were performed at least three times, and mean values and standard deviation were calculated.

\section{Results}

Curcumin inhibits proliferation of NPC cells in a dose- and time-dependent manner. To determine whether curcumin may regulate the proliferation of NPC cells, we examined the effect of curcumin on cell proliferation in two human NPC cell lines, 
A
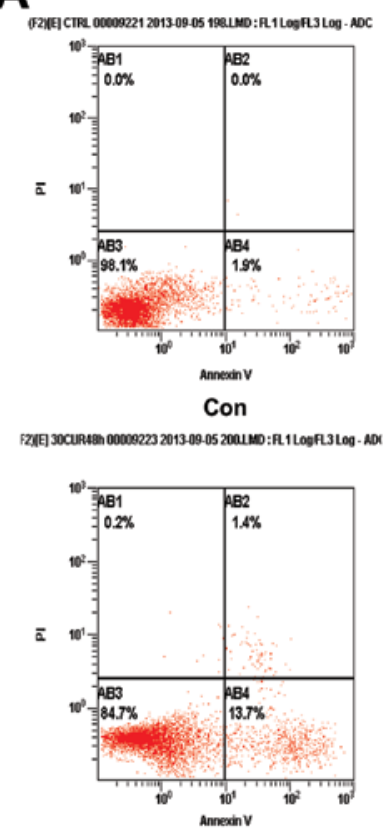

Curcumin $30 \mu \mathrm{M}$

B
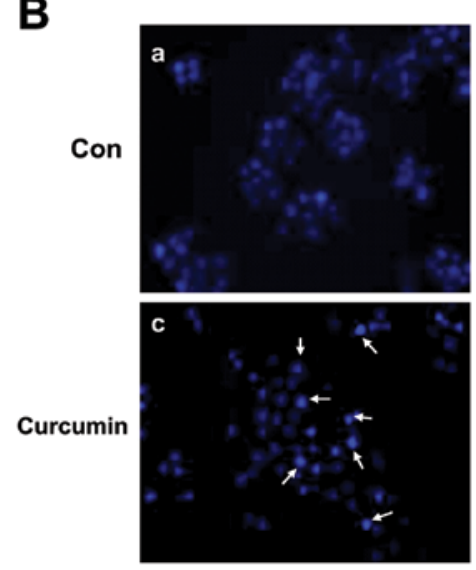

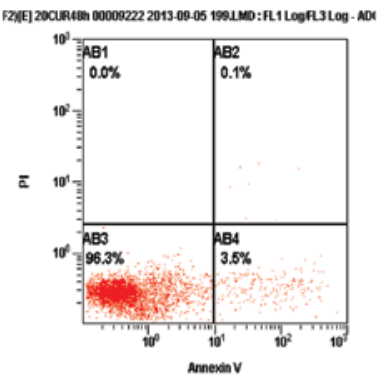

Curcumin $20 \mu \mathrm{M}$
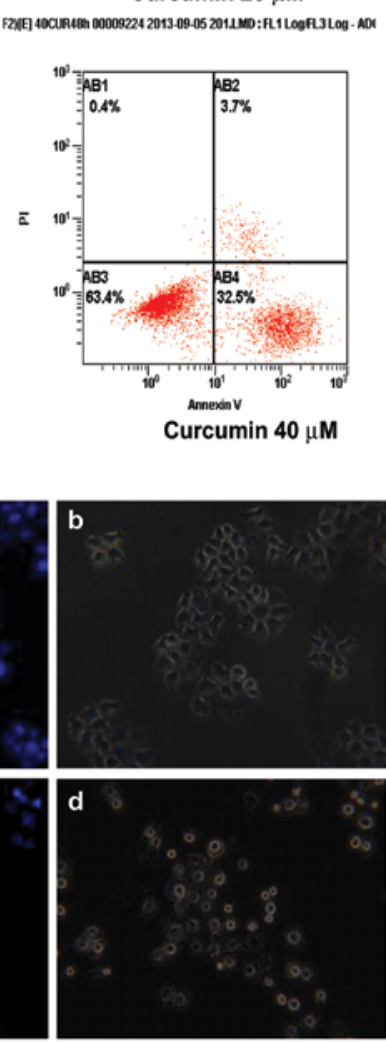

Figure 2. Curcumin induces apoptosis in CNE2 cells. (A) CNE2 cells were incubated with indicated doses of curcumin for 48 h, followed by staining with

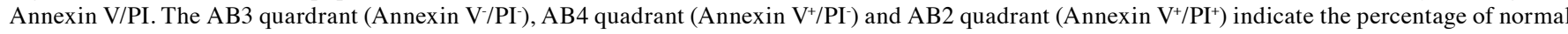
cells, early apoptosis and late apoptosis, respectively. (B) Apoptotic nuclear morphology changes induced by curcumin (40 $\mu \mathrm{M})$ treatment for $48 \mathrm{~h}$ were observed by Hoechst 33258 staining in CNE2 cells. Panels a and c show Hoechst 33258 nuclear staining, panels b and d show cell morphology. Arrows in panel $\mathrm{c}$ indicate chromatin condensation and nuclear fragmentation. The data are presented as mean $\pm \mathrm{SD}$ of three separate experiments. * $\mathrm{P}<0.05$ indicates significant difference as compared to the untreated control group.

CNE2 and HNE2, by a MTT assay. The results showed that treatment with increased concentrations of curcumin for up to $72 \mathrm{~h}$ significantly inhibited cell proliferation in a dose- and time-dependent manner (Fig. 1A and B).

Curcumin increases apoptosis of CNE2 cells. We evaluated the effects of curcumin on apoptosis in CNE2 cells using Annexin V-FITC and PI staining. In normal live cells, phosphatidy serine (PS) was located on the cytoplasmic surface of the cell membrane. However, in the apoptotic cells, PS was found to be translocated from the inner to the outer leaflet of the plasma membrane, thus exposing PS to the external cellular environment. The human anticoagulant, Annexin V, is a $35-36 \mathrm{Kda} \mathrm{Ca}^{+}$-dependent phospholipid binding protein
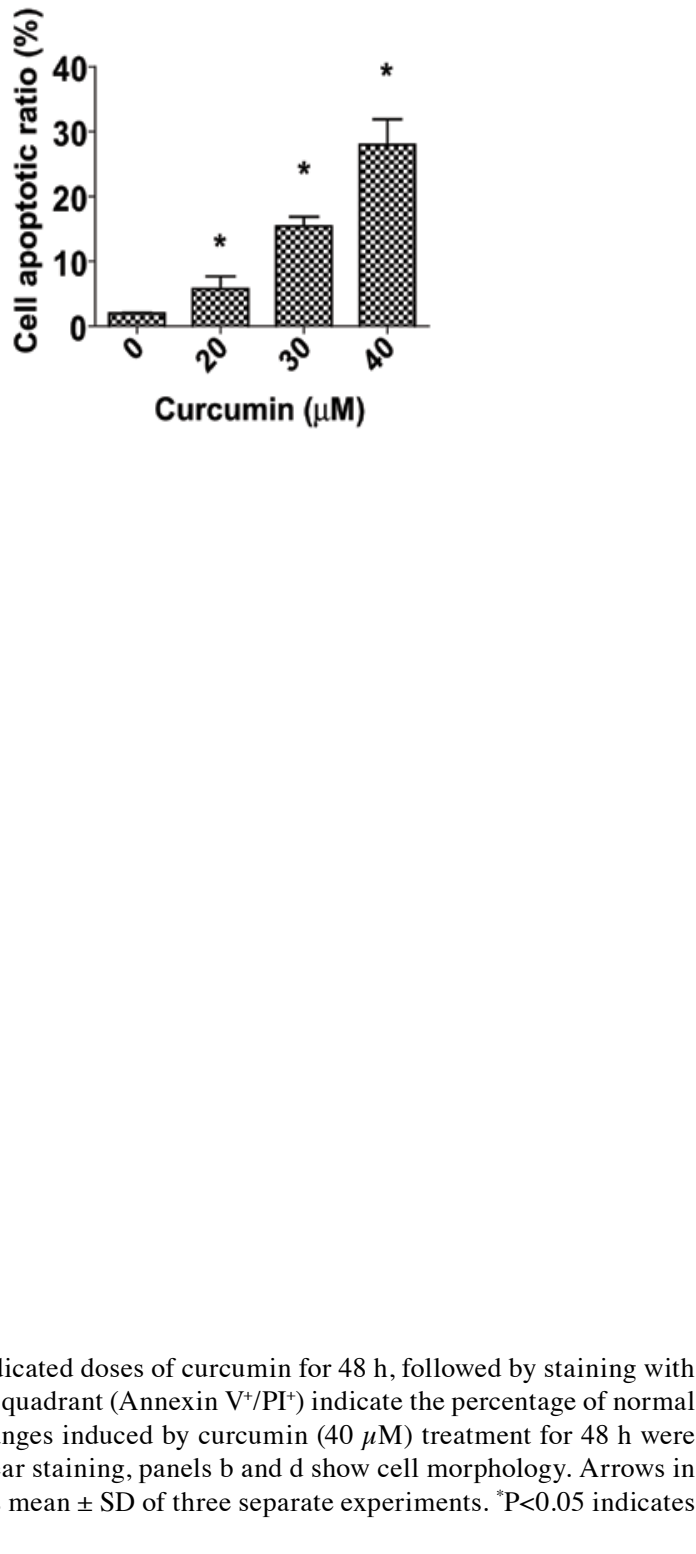

that has a high affinity for PS. Annexin V labeled with a fluorophore or biotin can identify apoptotic cells. In the scatter plot of double variable flow cytometry, AB3 quadrant (FITC $/ \mathrm{PI}^{-}$) showed living cells; $\mathrm{AB} 2$ quadrant $\left(\mathrm{FITC}^{+} / \mathrm{PI}^{+}\right)$stand for late apoptotic cells; and $\mathrm{AB} 4$ quadrant $\left(\mathrm{FITC}^{+} / \mathrm{PI}^{-}\right)$represents early apoptotic cells. As shown in Fig. 2A, a marked dose-dependent increase in both the early and late stages of apoptosis was observed in CNE2 cells after curcumin treatment compared to the control cells.

Morphological changes in the apoptotic cells were revealed by the Hoechst 33258 staining ( Fig. 2B). In the untreated CNE2 cells, the nuclei were stained weakly, and homogeneously blue, whereas, in cells treated with curcumin some bright chromatin condensation and nuclear fragmentation were observed. 
CNE2

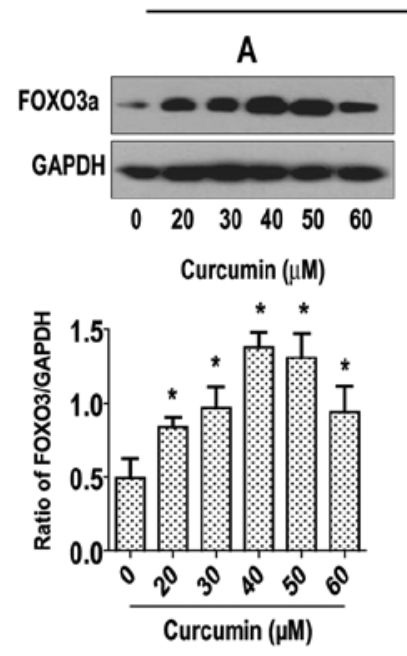

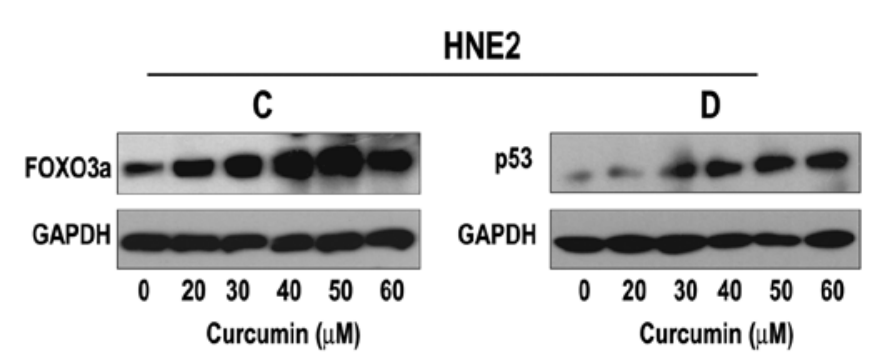
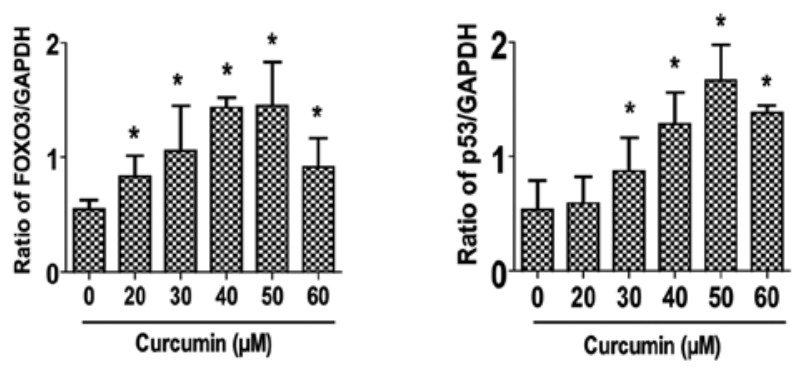

E

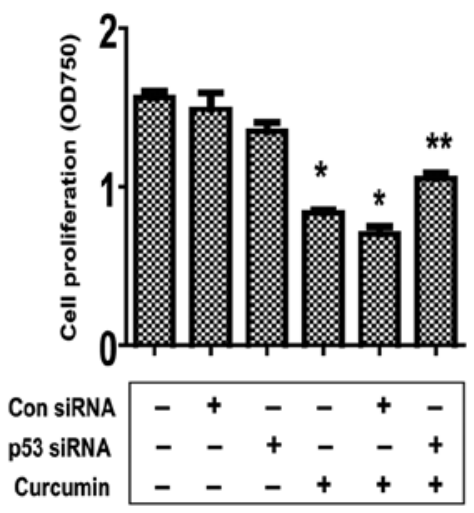

$\mathbf{F}$

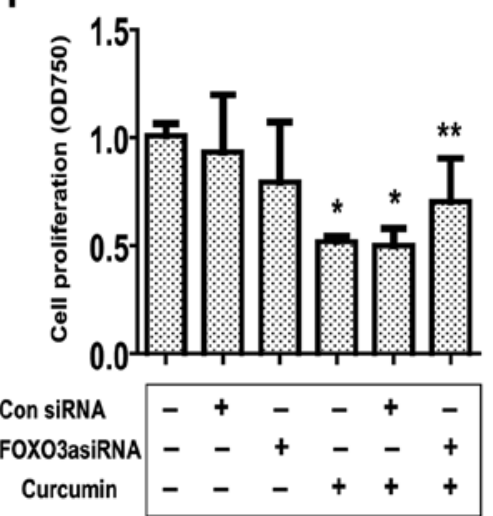

Figure 3. Curcumin increased FOXO3a and p53 protein expression; silencing of FOXO3a and p53 blocked the inhibitory effect of curcumin on cell growth. (A and B) Human CNE2 cells were treated with induced concentrations of curcumin as indicated for $24 \mathrm{~h}$. The expression of (A) FOXO3a and (B) p53 protein were detected by western blot analysis. (C and D) Human HNE2 cells were treated with induced concentrations of curcumin as indicated for 24 h. The expression of (C) FOXO3a and (D) p53 protein were detected by western blot analysis. GAPDH was used as an internal control for loading purpose. The graphs are the densitometry results of the FOXO3a and p53 protein expression over GAPDH in at least three separate experiments. (E and F) Human CNE2 cells were transfected with control or (E) p53 or (F) FOXO3a siRNAs (100 nM each) for $24 \mathrm{~h}$ before exposure to curcumin (40 $\mu \mathrm{M}$ ) for an additional $24 \mathrm{~h}$. Cell proliferation was determined by MTT assays. The data are presented as mean \pm SD of three separate experiments. * $<<0.01$ indicates significant difference from untreated control cells. ${ }^{* *} \mathrm{P}<0.05$ indicates significance of combination treatment as compared with curcumin alone.

Curcumin increases FOXO3a and p53 protein expression; silencing of $\mathrm{FOXO} 3 a$ and p53 abrogates the inhibitory effect of curcumin on growth of NPC cells. To elucidate the potential molecular mechanism underlying this effect, we tested the effect of curcumin on the expression of p53 and FOXO3a active proteins, these tumor suppressors have been shown to be involved in cell proliferation and apoptosis $(5,12)$. As shown in Fig. 3A and B, curcumin induced the protein expression of both FOXO3a (Fig. 3A) and p53 (Fig. 3B) dosedependently in CNE2 cells, with maximal induction observed at $40-50 \mu \mathrm{M}$, respectively. Note that similar results were also observed in an additional cell line (HNE2) (Fig. 3C and D).

To further explore the role of these tumor suppressors in mediating the curcumin-inhibited NPC cell proliferation, we blocked p53 and FOXO3a gene expression by transfecting CNE2 cells with p53 and FOXO3a siRNAs and evaluated the effects of curcumin on cell proliferation. We demonstrated that the knockdown of p53 and FOXO3a genes significantly overcame the curcumin-mediated growth inhibition of CNE2 cells (Fig. 3E). This indicated important roles of p53 and FOXO3a expression in curcumin-inhibited cell growth.

Curcumin induces ERK/MAPK activation, blockade of ERK 1/2 abrogated the effect of curcumin on FOXO3a and p53 expression, while restoring the cell growth in the presence of curcumin in NPC cells. In order to determine the potential signaling pathways that are involved in the curcumin-mediated regulation of FOXO3a and p53 expression, thereby controlling cell proliferation, we initially evaluated the effect of curcumin on the activation of ERK and p38 MAPK by western blot analysis. As shown in Fig. 4A, treatment with curcumin at $40 \mu \mathrm{M}$ significantly increased the phosphorylation of ERK1/2 starting at $30 \mathrm{~min}$ and continued for up to $24 \mathrm{~h}$, whereas the level of total ERK1/2 protein was unchanged. However, curcumin had little effect on the activation and protein expression of p38 MAPK. Similar results 

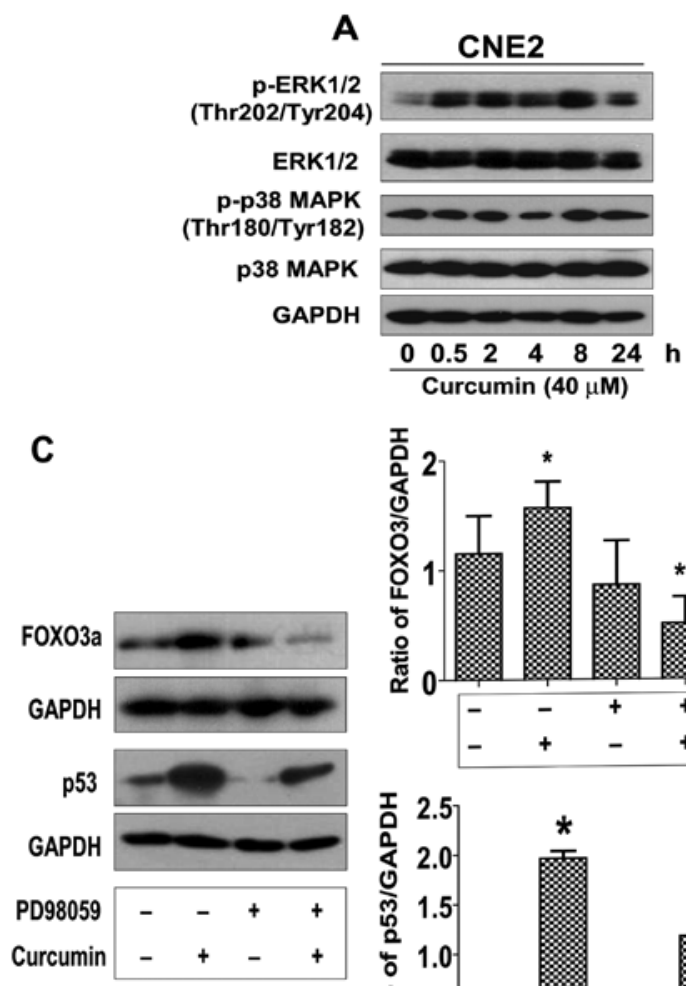
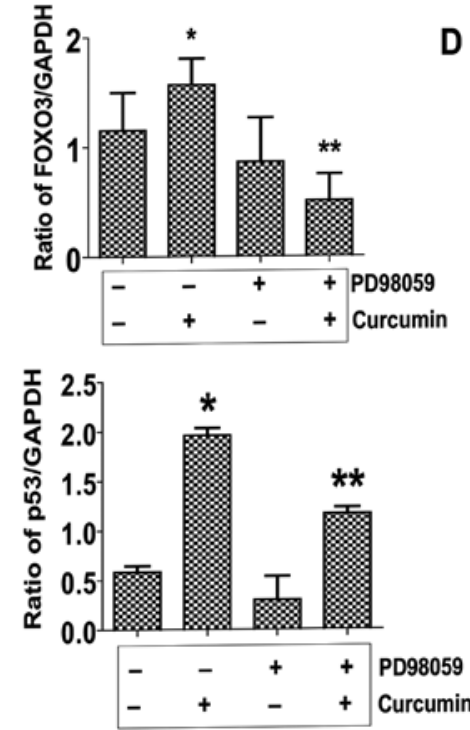

D
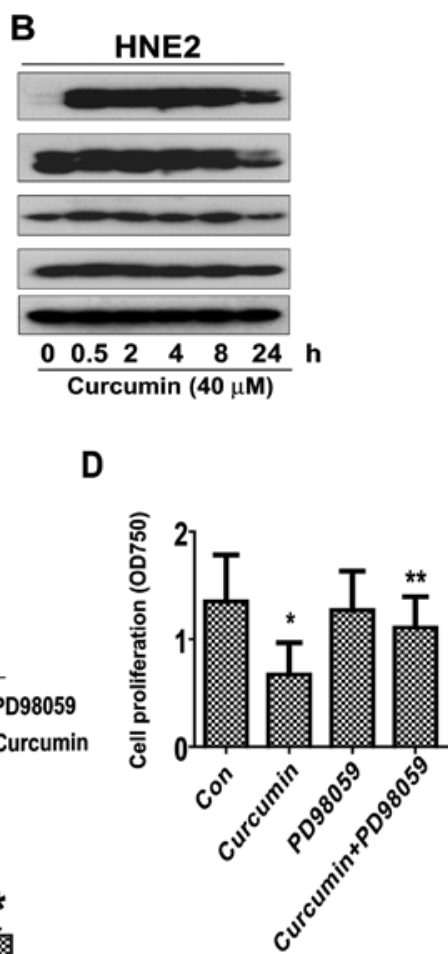

Figure 4. Curcumin induces ERK/MAPK activation, blockade of ERK1/2 abrogates the effect of curcumin on FOXO3a and p53 expression, while restoring the cell growth in the presence of curcumin in NPC cells. (A and B) Human CNE2 and HNE2 cells were treated with curcumin at the concentration of $40 \mu \mathrm{M}$ for up to $24 \mathrm{~h}$. The expression of the phosphorylated or total protein of ERK1/2, p38 MAPK were measured by western blot analysis using corresponding antibodies. GAPDH was used as loading control. (C) Human CNE2 cells were treated with PD98059 for $2 \mathrm{~h}$, followed by exposure to curcumin (40 $\mu \mathrm{M}$ ) for an additional $24 \mathrm{~h}$. The expression of p53 and FOXO3a proteins were detected by western blot analysis using specific antibodies. The bar graphs represent the densitometry results of $\mathrm{p} 53$ and FOXO3a/GAPDH as mean \pm SD of at least three separate experiments. (D) Human CNE2 cells were treated with PD98059 for $2 \mathrm{~h}$, followed by exposure to curcumin $(40 \mu \mathrm{M})$ for an additional $24 \mathrm{~h}$. Cell proliferation was determined by MTT assays. The data are presented as mean $\pm \mathrm{SD}$ of three separate experiments. ${ }^{*} \mathrm{P}<0.01$ indicates significant difference from untreated control cells. ${ }^{* *} \mathrm{P}<0.01$ indicates significance of combination treatment as compared with curcumin alone.

were also observed in an additional NPC cell line (HNE2) (Fig. 4B). Interestingly, we showed that the ERK inhibitor (PD98059) exposed to the cells abolished the curcumininduced FOXO3a and p53 protein expression in CNE2 cells (Fig. 4C). Moreover, treatment of PD98059 restored the cell growth in the presence of curcumin (Fig. D). Together, the above results suggested that activation of ERK signaling pathway was critical in mediating the effect of curcumin on regulating FOXO3a and p53 expression, and cell growth.

p53 is upstream of FOXO3a; exogenous expression of FOXO3a restores the effect of curcumin on growth of cells silenced by endogenous FOXO3a gene. Transcriptional activity of FOXO3a could be modulated though interactions with other transcriptional factors such as tumor suppressor p53 (15), thus, we assessed if curcumin affected the interactions of FOXO3a with p53. As shown in Fig. 5, silencing and blockade of p53 using either siRNA or specific p53 inhibitor overcame the induced effect of curcumin on the expression of FOXO3a protein (Fig. 5A and B). On the contrary, silencing or overexpression of FOXO3a had no effect on curcumin-induced p53 protein expression (Fig. $5 \mathrm{C}$ and D), but strengthened the inhibitory effect of curcumin on cell growth (Fig. 5E). Interestingly, exogenous expression of FOXO3a restored the effect of curcumin on growth of cells silenced by endogenous FOXO3a gene (Fig. 5F). Note that western blot analysis confirmed the exogenous expression of FOXO3a protein transfected into the cells (Fig. 5D-F).

\section{Discussion}

Studies have shown that curcumin can inhibit the growth of a variety of tumor cells, as well as induce cell apoptosis in several tumors including NPC cells (16-20) suggesting that curcumin can be used as a natural antitumor agent. However, the detailed mechanisms by which this agent targets NPC cancer cells remained unclear. In this study, we evaluated the response of NPC cells to curcumin treatment. Our results indicated that curcumin inhibited NPC cell proliferation and induced apoptosis in a dose- and time-dependent manner suggesting a tumor suppressor property of this agent. Of note, the concentrations of curcumin used here were consistent with 
A

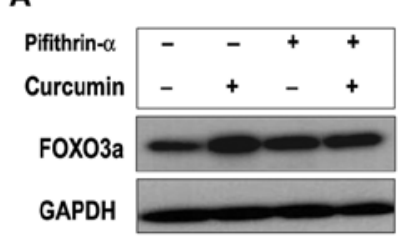

B

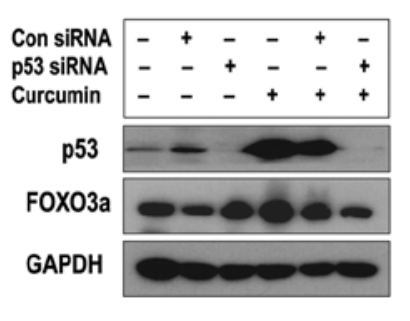

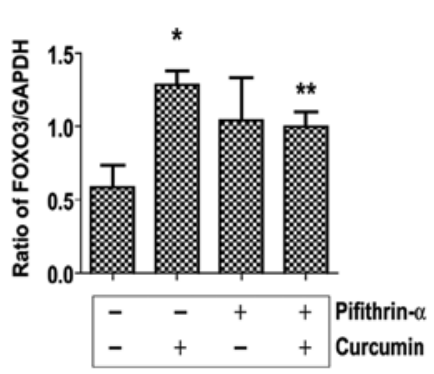

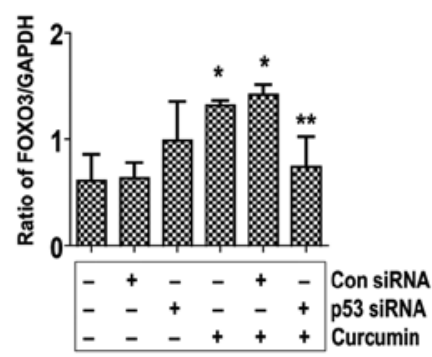

C

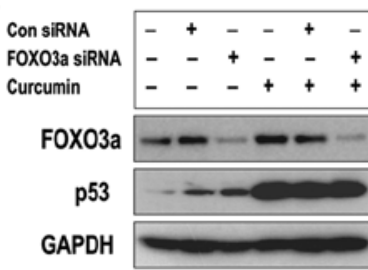

D

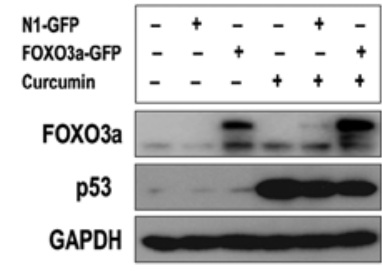

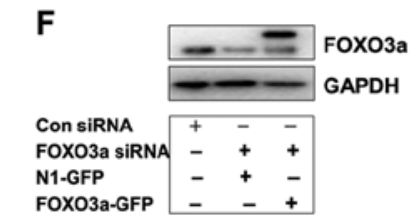

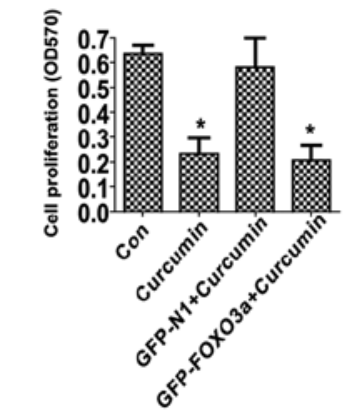

$\mathbf{F}$
E
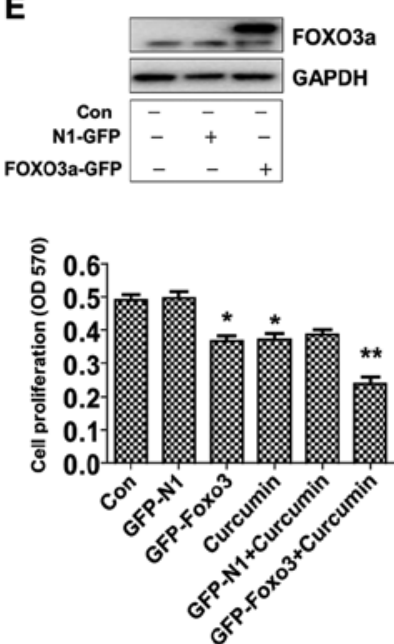
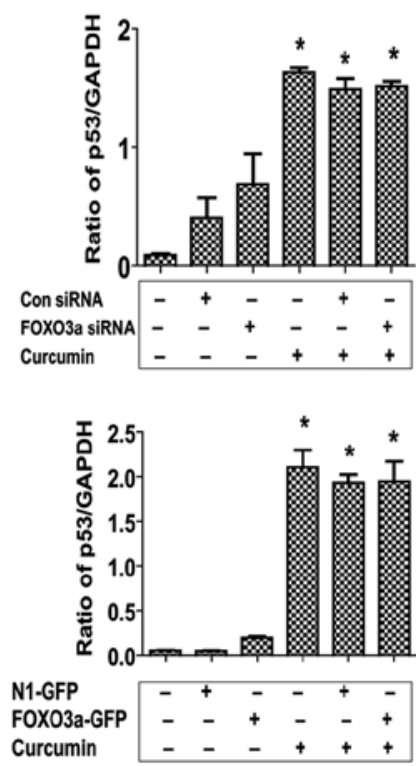

Figure 5. p53 is upstream of FOXO3a. Exogenous expression of FOXO3a restored the effect of curcumin on growth of cell silenced by endogenous $\mathrm{FOXO3a}$ gene. (A) CNE2 cells were treated with p53 inhibitor Pifithrin- $\alpha(10 \mu \mathrm{M})$ for $2 \mathrm{~h}$, followed by exposure to curcumin $(40 \mu \mathrm{M})$ for an additional $24 \mathrm{~h}$. The expression of p53 and FOXO3a was detected by western blot analysis. (B) CNE2 cells were transfected with control or p53 siRNAs (100 nM) for $24 \mathrm{~h}$, followed by exposure to curcumin $(40 \mu \mathrm{M})$ for an additional $24 \mathrm{~h}$. The expression of $\mathrm{p} 53$ and FOXO3a protein was detected by western blot analysis. (C) CNE2 cells were transfected with control or FOXO3a siRNAs (100 nM each) for $24 \mathrm{~h}$ before exposure to curcumin $(40 \mu \mathrm{M})$ for an additional $24 \mathrm{~h}$. The expression of p53 was detected by western blot analysis. (D) CNE2 cells were transfected with control (pEGFP-N1) or FOXO3a expression vector (FOXO3a-pEGFP) for $24 \mathrm{~h}$, and then treated with curcumin $(40 \mu \mathrm{M})$ for an additional $24 \mathrm{~h}$. The expression of p53 and FOXO3a protein was detected by western blot analysis. (E) CNE2 cells were transfected with control (pEGFP-N1) or FOXO3a expression vector (FOXO3a-pEGFP) for $24 \mathrm{~h}$ before exposure to curcumin (40 $\mu \mathrm{M}$ ) for an additional $24 \mathrm{~h}$. (F) CNE2 cells were transfected with control or FOXO3a siRNA for $30 \mathrm{~h}$, followed by control (pEGFP-N1) or FOXO3a expression vector (FOXO3a-pEGFP) for up to $24 \mathrm{~h}$ before exposure to curcumin $(40 \mu \mathrm{M})$ for an additional $24 \mathrm{~h}$. Cell proliferation was determined by MTT assays. The upper insert panels are blots of expression of FOXO3a protein detected by western blot analysis. ${ }^{\text {"P }}<0.01$ indicates significant difference from untreated control cells. ${ }^{* *} \mathrm{P}<0.05$ indicates significance of combination treatment as compared with curcumin alone.

or even lower then those reported by others demonstrating significant responses in different cell systems (21-23) although lower doses were also reported in other studies $(24,25)$. We realized that a higher dose was needed to inhibit different cancer cell growth, but this was within the range of those reported by others and showed no toxicity (21-23).

In our study we demonstrated the role of p53 and FOXO3a protein induction that mediated the effect of curcumin on the inhibition of NPC cell growth. As tumor suppressors, both transcription factors play important roles in several areas including gene regulation, cell growth and apoptosis $(5,12)$. Striking similarities have been reported between p53 and
FOXO, such as post-translational modifications, common signaling pathways, target genes, and similar mutual interactions with various proteins (26). We found that blockade of the activity of p53 or silencing of either p53 or FOXO3a gene partially overcame the inhibitory effect of curcumin on NPC cell proliferation, suggesting that induction of these two molecules contributed to mediation of the effect of curcumin on NPC cell growth inhibition. Whether curcumin affected the post-translational modifications, such as phosphorylation, acetylation and ubiquitination, of either p53 or FOXO3a, thereby regulating their subcellular localization and transcriptional activities require further study. Consistent with 
this, other studies also found the link of curcumin and p53 or FOXO3a expression, and demonstrated the role of these transcription factors in mediating the effect of curcumin on controlling cell proliferation and other functions in other cell systems $(27,28)$. We reasoned that more studies are required to explore the precise mechanism of p53 and FOXO3a expression, regulation and downstream pathways in mediating the overall response of curcumin.

The MAPK signaling pathway plays a key role in the regulation of gene expression, cellular growth and survival (29). Data from others indicated that curcumin activates MAPK signaling pathways, and that activation of MAPK, such as ERK and p38 MAPK, links curcumin-mediated signaling to the transcriptional regulation of genes that are crucial for cell growth inhibition $(30,31)$. Our result identified an important role of ERK activation in mediating the inhibitory effect of curcumin on p53 and FOXO3a protein expression and NPC cell growth inhibition. However, p38 MAPK played no role in our study, which differed from others $(31,32)$. The discrepancy remain unclear, the use of different cell lines and the culture conditions may account for this, which needs to be further determined. We reasoned that curcumin may function through activating ERK/MAPK signaling pathway, followed by upregulation of p53 and FOXO3a protein expression, thereby inhibiting NPC cell proliferation and inducing apoptosis. However, inhibition of ERK/MAPK reversed the effect of curcumin on NPC proliferation and could be independent of p53 and FOXO3a signaling. Also, the potential downstream targets of p53 and FOXO3a may be involved in this process. Thus, more studies are required to further elucidate this.

The aforementioned uncovered that both p53 and FOXO3a played a causative role in mediating the effect of curcumin in controlling NPC cell growth and perhaps inducing apoptosis. Furthermore, our results showed that exogenous expression of FOXO3a could enhance the effect of curcumin on cell growth, and more importantly, restore the inhibitory effect of curcumin on growth of NPC cells silenced by endogenous FOXO3a gene in siRNA approach, while having no further effect on curcumin-induced p53 expression. This further confirmed the role of FOXO3a in mediating the effect of curcumin on cell growth; it also suggested that p53 is upstream of FOXO3a and induction of FOXO3a by curcumin is p53-dependent. We speculated that p53 and FOXO may have parallel functions that worked in concert to mediate the effect of curcumin on control growth of NPC cells. The p53 and FOXO3a interaction have been identified (26), however, the extent and importance of the functional interaction between these two have not been fully explored. Consistent with our report, other studies found that FOXO3a was a p53 target gene and that transcriptional activity of FOXO3a was regulated by $\mathrm{p} 53$, while the latter was not affected by FOXO3a $(33,34)$. The p53 and FOXO3a formed part of regulation transcriptional network to control cancer cell growth and apoptosis $(33,34)$. In addition, curcumin induced expression of p53 or/and FOXO3a in inhibition of cancer cell growth and other functions have been shown in other cell systems $(27,28,35,36)$. However, whether curcumin affects the interaction of these proteins to facilitate the inhibitory effect of NPC growth remain to be determined. A number of signaling pathways mediated the expression of $\mathrm{FOXO} 3 \mathrm{a}$ and p53, and critically regulated apoptotic events, which included PI3-K/Akt and cyclin-dependent kinase (CDK) $(37,38)$. We believed that understanding the functional interactions of $\mathrm{p} 53$ and FOXO3a, their regulated signaling pathways and known downstream targets will be crucial to better elucidate their roles in mediating the overall response of curcumin.

In summary, our data show that curcumin inhibits the growth and induces apoptosis of NPC cells through activation of the ERK signaling pathway; this leads to increase in protein expression of p53 and FOXO3a. p53 is upstream of FOXO3a and induction of FOXO3a by curcumin is p53-depedent. Both transcription factors and tumor suppressors form a regulatory loop that work in concert to mediate the effect of curcumin. This study unveils a new mechanism by which curcumin inhibits the proliferation and induces apoptosis of NPC cells.

\section{Acknowledgements}

This study was supported in part by the Research Fund from Guangdong Province Administration of Traditional Chinese Medicine (20132149), the Special Science and Technology Join fund from Guangdong Provincial Department of Science and Technology-Guangdong Academy of Traditional Chinese Medicine (2012A032500011) and a grant from the National Nature Scientific Foundation of China (81272614).

\section{References}

1. Xu Y, Zhang J, Shi W and Liu Y: Anticancer effects of 3,3'-diindolylmethane are associated with G1 arrest and mitochondria-dependent apoptosis in human nasopharyngeal carcinoma cells. Oncol Lett 5: 655-662, 2013.

2. Pan Y, Zhou F, Zhang R and Claret FX: Stat3 inhibitor Stattic exhibits potent antitumor activity and induces chemo- and radio-sensitivity in nasopharyngeal carcinoma. PloS One 8: e54565, 2013.

3. Aggarwal BB, Sundaram C, Malani N and Ichikawa H: Curcumin: the Indian solid gold. Adv Exp Med Biol 595: 1-75, 2007.

4. Wilken R, Veena MS, Wang MB and Srivatsan ES: Curcumin: A review of anti-cancer properties and therapeutic activity in head and neck squamous cell carcinoma. Mol Cancer 10: 12, 2011.

5. Nayak G and Cooper GM: p53 is a major component of the transcriptional and apoptotic program regulated by PI 3-kinase/ Akt/GSK3 signaling. Cell Death Dis 3: e400, 2012.

6. Hollstein M, Sidransky D, Vogelstein B and Harris CC: p53 mutations in human cancers. Science 253: 49-53, 1991.

7. Zeng GQ, Yi H, Li XH, et al: Identification of the proteins related to p53-mediated radioresponse in nasopharyngeal carcinoma by proteomic analysis. J Proteomics 74: 2723-2733, 2011.

8. Shen YA, Lin $\mathrm{CH}$, Chi $\mathrm{WH}$, et al: Resveratrol impedes the stemness, epithelial-mesenchymal transition, and metabolic reprogramming of cancer stem cells in nasopharyngeal carcinoma through p53 activation. Evid Based Complement Alternat Med 2013: 590393, 2013.

9. Brunet A, Bonni A, Zigmond MJ, et al: Akt promotes cell survival by phosphorylating and inhibiting a Forkhead transcription factor. Cell 96: 857-868, 1999.

10. Schmidt M, Fernandez de Mattos S, van der Horst A, et al: Cell cycle inhibition by FoxO forkhead transcription factors involves downregulation of cyclin D. Mol Cell Biol 22: 7842-7852, 2002.

11. Obsil T and Obsilova V: Structural basis for DNA recognition by FOXO proteins. Biochim Biophys Acta 1813: 1946-1953, 2011.

12. Yang JY, Zong CS, Xia W, et al: ERK promotes tumorigenesis by inhibiting FOXO3a via MDM2-mediated degradation. Nat Cell Biol 10: 138-148, 2008. 
13. Shou Z, Lin L, Liang J, Li JL and Chen HY: Expression and prognosis of FOXO3a and HIF-1alpha in nasopharyngeal carcinoma. J Cancer Res Clin Oncol 138: 585-593, 2012.

14. Wen Q, Duan X, Liao R, et al: Characterization of intracellular translocation of Forkhead transcription factor O (FoxO) members induced by NGF in PC12 cells. Neurosci Lett 498: 31-36, 2011.

15. Wang F, Marshall CB, Yamamoto K, et al: Biochemical and structural characterization of an intramolecular interaction in FOXO3a and its binding with p53. J Mol Biol 384: 590-603, 2008.

16. Ye F, Zhang GH, Guan BX and Xu XC: Suppression of esophageal cancer cell growth using curcumin, (-)-epigallocatechin-3-gallate and lovastatin. World J Gastroenterol 18 : $126-135,2012$.

17. Subramaniam D, Ponnurangam S, Ramamoorthy $\mathrm{P}$, et al: Curcumin induces cell death in esophageal cancer cells through modulating Notch signaling. PloS One 7: e30590, 2012.

18. Quitschke WW: Curcuminoid binding to embryonal carcinoma cells: reductive metabolism, induction of apoptosis, senescence, and inhibition of cell proliferation. PloS One 7: e39568, 2012.

19. Sahu RP, Batra S and Srivastava SK: Activation of ATM/Chk1 by curcumin causes cell cycle arrest and apoptosis in human pancreatic cancer cells. Br J Cancer 100: 1425-1433, 2009.

20. Bao B, Ali S, Banerjee S, et al: Curcumin analogue CDF inhibits pancreatic tumor growth by switching on suppressor microRNAs and attenuating EZH2 expression. Cancer Res 72: 335-345, 2012.

21. Chen CC, Sureshbabul M, Chen HW, et al: Curcumin suppresses metastasis via Sp-1, FAK inhibition, and E-cadherin upregulation in colorectal cancer. Evid Based Complement Alternat Med 2013: 541695, 2013.

22. Wang D, Hu J, Lv L, Xia X, Liu J and Li X: Enhanced inhibitory effect of curcumin via reactive oxygen species generation in human nasopharyngeal carcinoma cells following purple-light irradiation. Oncol Lett 6: 81-85, 2013.

23. Sundram V, Chauhan SC, Ebeling M and Jaggi M: Curcumin attenuates beta-catenin signaling in prostate cancer cells through activation of protein kinase D1. PloS One 7: e35368, 2012.

24. Jiang M, Huang O, Zhang X, et al: Curcumin induces cell death and restores tamoxifen sensitivity in the antiestrogen-resistant breast cancer cell lines MCF-7/LCC2 and MCF-7/LCC9. Molecules 18: 701-720, 2013.

25. Blakemore LM, Boes C, Cordell $\mathrm{R}$ and Manson MM Curcumin-induced mitotic arrest is characterized by spindle abnormalities, defects in chromosomal congression and DNA damage. Carcinogenesis 34: 351-360, 2013.
26. You $\mathrm{H}$ and Mak TW: Crosstalk between p53 and FOXO transcription factors. Cell Cycle 4: 37-38, 2005.

27. Qiao Q, Jiang Y and Li G: Curcumin enhances the response of non-Hodgkin's lymphoma cells to ionizing radiation through further induction of cell cycle arrest at the $\mathrm{G} 2 / \mathrm{M}$ phase and inhibition of mTOR phosphorylation. Oncol Rep 29: 380-386, 2013.

28. Singh AT, Ghosh M, Forte TM, Ryan RO and Gordon LI Curcumin nanodisk-induced apoptosis in mantle cell lymphoma. Leuk Lymphoma 52: 1537-1543, 2011.

29. Sebolt-Leopold JS, Herrera R and Ohren JF: The mitogenactivated protein kinase pathway for molecular-targeted cancer treatment. Recent Results Cancer Res 172: 155-167, 2007.

30. Li R, Wang Y, Liu Y, et al: Curcumin inhibits transforming growth factor-beta1-induced EMT via PPARgamma pathway, not Smad pathway in renal tubular epithelial cells. PloS One 8: e58848, 2013.

31. Kang D, Park W, Lee S, Kim JH and Song JJ: Crosstalk from survival to necrotic death coexists in DU-145 cells by curcumin treatment. Cell Signal 25: 1288-1300, 2013.

32. Wang WZ, Li L, Liu MY, et al: Curcumin induces FasL-related apoptosis through p38 activation in human hepatocellular carcinoma Huh7 cells. Life Sci 292: 352-358, 2013.

33. Renault VM, Thekkat PU, Hoang KL, et al: The pro-longevity gene FoxO3 is a direct target of the p53 tumor suppressor. Oncogene 30: 3207-3221, 2011.

34. Miyaguchi Y, Tsuchiya K and Sakamoto K: P53 negatively regulates the transcriptional activity of FOXO3a under oxidative stress. Cell Biol Int 33: 853-860, 2009.

35. Guo LD, Chen XJ, Hu YH, Yu ZJ, Wang D and Liu JZ: Curcumin inhibits proliferation and induces apoptosis of human colorectal cancer cells by activating the mitochondria apoptotic pathway. Phytother Res 27: 422-430, 2013.

36. Zingg JM, Hasan ST, Cowan D, Ricciarelli R, Azzi A and Meydani M: Regulatory effects of curcumin on lipid accumulation in monocytes/macrophages. J Cell Biochem 113: 833-840, 2012.

37. Tudzarova S, Trotter MW, Wollenschlaeger A, et al: Molecular architecture of the DNA replication origin activation checkpoint. EMBO J 29: 3381-3394, 2010.

38. Amente S, Zhang J, Lavadera ML, Lania L, Avvedimento EV and Majello B: Myc and PI3K/AKT signaling cooperatively repress FOXO3a-dependent PUMA and GADD45a gene expression. Nucleic Acids Res 39: 9498-9507, 2011. 